BULL. AUSTRAL. MATH. SOC.

VOL. 12 (1975), 27-30.

\title{
A note on the edge reconstruction conjecture
}

\section{E.F. Schmeichel}

Let $G$ be a graph with vertex degree sequence

$d_{1} \leq d_{2} \leq \ldots \leq d_{p}$. It is shown that if $d_{i}+d_{p-i+1} \geq p$ for

some $i$, then $G$ is uniquely reconstructable from its

collection of maximal (edge deleted) subgraphs. This generalizes considerably a result of Lovász. As a corollary, it is shown that Chvátal's existence condition for hamiltonian cycles implies edge reconstructability as well.

In this paper, we consider only finite, undirected graphs without loops or multiple edges. We will denote the set of vertices and edges of a graph $G$ by $V(G)$ and $E(G)$, respectively.

Kelly [4] and Ulam [6, p. 29] proposed the following interesting conjecture.

VERTEX RECONSTRUCTION CONJECTURE. Any graph with at least three vertices can be uniquely reconstruated from its colleation of maximal induced subgraphs $G-v, v \in V(G)$.

The following related conjecture was formulated by Harary [3].

EDGE RECONSTRUCTION CONJECTURE. Any graph with at least four edges can be uniquely reconstructed from its collection of maximal subgraphs $G-e, e \in E(G)$.

The edge conjecture has been proven weaker than the vertex conjecture, but even so seems quite difficult. In [5], Lovász ingeniously proved that

Received 18 September 1974. 
the edge conjecture was true for any graph with more edges than its complement. The purpose of this note is to generalize Lovász's result; the proof will be based on the technique given in [5].

Recall [2, Lemma 1.4] that the collection of maximal subgraphs $G-e, e \in E(G)$, determines the vertex degree sequence of $G$. Our main result is then the following

THEOREM. Let $G$ be a graph with vertex degree sequence $d_{1} \leq d_{2} \leq \ldots \leq d_{p}$. If

$$
d_{i}+d_{p-i+1} \geq p
$$

for some $i$, then the edge reconstruction conjecture is true for $G$.

Proof. Suppose $B$ is another graph satisfying (1) and having the same collection of maximal subgraphs as $G$. If $G \rightarrow H$ denotes the set of monomorphisms of $G$ into $H$, we have by the familiar sieve formula (see, for example, Lovász [5]),

$$
|G \rightarrow H|=\sum_{X \subseteq G}(-1)^{|E(X)|}|X \rightarrow \bar{H}|,
$$

where $\bar{H}$ denotes the complement of $H$, and $X$ is taken over all subgraphs with $V(X)=V(G)$ and $E(X) \subseteq E(G)$. We also have

$$
|H \rightarrow H|=\sum_{X \subseteq B}(-1)^{\mid E(X)}|| X \rightarrow \bar{H} \mid .
$$

Since the hypothesis on the maximal subgraphs assures us that $G$ and $H$ have the same proper subgraphs (see [2, Lemma 1.2]), the terms of (2) and (3) will be the same for $X \neq G$ and $X \neq H$. Therefore if we could show that (1) implies $|G \rightarrow \bar{H}|=|H \rightarrow \bar{H}|=0$, it would follow that $|G \rightarrow H|=|H \rightarrow H|>0$, and $G$ and $H$ would be isomorphic. Hence $G$ would be uniquely reconstructable as asserted.

Note that the graph $\bar{H}$ will have vertex degree sequence $d_{1}^{\prime} \leq d_{2}^{\prime} \leq \ldots \leq d_{p}^{\prime}$, where $d_{i}^{\prime}=(p-1)-d_{p-i+1}$. So (1) means precisely that $d_{i}>d_{i}^{\prime}$ for some $i$. But the degree of an image vertex under a monomorphism must be at least as large as the degree of its corresponding preimage vertex. Hence if $d_{i}>d_{i}^{\prime}$ for some $i$, it would be impossible 
to map the vertices of degree greater than or equal to $d_{i}$ in $G$ (or $H$ ) monomorphically into $\bar{H}$, since at least one such vertex would have to be mapped into a vertex of smaller degree in $\bar{H}$. Thus (1) does imply $|G \rightarrow \bar{H}|=|H \rightarrow \bar{H}|=0$, and the proof of the theorem is complete.

COROLLARY 1 (Lovász). If $G$ contains more edges than its complement, the edge reconstruction conjecture is true for $G$.

For then the mean value of $d_{i}+d_{p-i+1}$ taken over all $i$ exceeds $p-1$, and hence $d_{i}+d_{p-i+1} \geq p$ for some $i$.

In [1], Chvátal showed that a graph with vertex degrees $d_{1} \leq d_{2} \leq \ldots \leq d_{p}$ satisfying

$$
d_{k} \leq k<\frac{p}{2} \text { implies } d_{p-k} \geq p-k
$$

is hamiltonian. As an interesting application of the theorem, we show that Chvátal's condition for hamiltonian cycles also guarantees edge reconstructability.

COROLLARY 2. A graph $G$ whose vertex degrees $d_{1} \leq d_{2} \leq \ldots \leq d_{p}$ satisfy (4) is edge reconstructable.

Proof. Note that $d_{1}$ - and hence $d_{2}$ - must be greater than or equal to 2 . If $d_{2}=2$, then $d_{p-2} \geq p-2$ by (4). This means (1) is satisfied for $i=2$, and $G$ is edge reconstructable by the theorem. Assuming $d_{2} \geq 3$, we also have $d_{3} \geq 3$. If $d_{3}=3$, then $d_{p-3} \geq p-3$ by (4). This means ( 1 ) is satisfied for $i=3$, and $G$ is edge reconstructable by the theorem. So we may assume $d_{3} \geq 4$. Continuing in this way, we obtain $d_{[p-1 / 2]} \geq[p+1 / 2]$. But then taking $i=[p+1 / 2]$, we find $d_{i}+d_{p-i+1} \geq 2[p+1 / 2] \geq p$, and $G$ would be edge reconstructable by the theorem.

\section{References}

[1] V. Chvátal, "On Hamilton's ideals", J. Combinatorial Theory Ser. B.12 (1972), 163-168. 
[2] D.L. Greenwell and R.L. Hemminger, "Reconstructing graphs", The many facets of graph theory, 91-114 (Lecture Notes in Mathematics, 110. Springer-Verlag, Berlin, Heidelberg, New York, 1969).

[3] F. Harary, "On the reconstruction of a graph from a collection of subgraphs", Theory of graphs and its applications, 47-52 (Proc. Sympos., Smolenice, June 1963. Publishing House of the Czechoslovak Academy of Sciences, Prague, 1964).

[4] Paul J. Kelly, "A congruence theorem for trees", Pacific J. Math. 7 $(1957), 961-968$.

[5] L. Lovász, "A note on the line reconstruction problem", $J$. Combinatorial Theory Ser. B 13 (1972), 309-310.

[6] S.M. Ulam, A collection of mathematical problems (Interscience, New York, London, 1960).

Department of Mathematics,

University of Southern California,

Los Angeles,

Cal ifornia,

USA. 